Multiresistenter Staphylococcus aureus bei Diabetikern

\title{
Generelles Screening würde Klarheit schaffen
}

\author{
Im diabetologischen Alltag ist MRSA ist in den vergangenen Jahren zu einem wichtigen \\ Thema geworden. Chronische Wunden bei Diabetikern sind prädestiniert für diese \\ Bakterien. Dessen sollte man sich beim Hygienemanagement ebenso bewusst sein, \\ wie bei der Verordnung von Antibiotika.
}

Die Zahl der Infektionen mit multiresistenten Staphylococcus aureus (MRSA) liegt in Deutschland bei etwa 180000 pro Jahr, 3000 davon enden tödlich. 25\% aller nosokomialer Infektionen in Deutschland werden durch MRSA verursacht. „Damit liegt Deutschland im europäischen Mittelfeld“, sagte Dr. Hans-Jörg Höning, niedergelassener Chirurg in Hamm. Während in südeuropäischen Ländern das MRSA-Problem noch sehr viel größer sei, verursacht das Bakterium in den Niederlanden nur 0,1\% der Krankenhausinfektionen. Entscheidend dafür ist, dass in den Niederlanden jeder Bürger auf MRSA gescreent wird und Antibiotika sehr viel restriktiver verordnet werden. „Die Niederländer kommen mit $10 \%$ weniger Antibiotikaverordnungen aus, ohne dass vermehrt schwere bakterielle Infektionen auftreten“, so Höning. Angesichts der aktuellen Entwicklung in Deutschland sei es sinnvoll, auch bei uns ein generelles konsequentes Screening einzuführen.

\section{Konsequentes Hygienemanagement}

Beim Umgang mit Patienten, bei denen eine Kolonisation mit MRSA vermutet wird oder nachgewiesen ist, erfordert ein konsequentes Hygienemanagement. Dazu gehört das Screening bei Risikopatienten ebenso wie die Isolierung besiedelter Patienten. Sehr wichtig ist die Händedesinfektion, die Benutzung von Handschuhen, Mundschutz und Schutzkittel. „Dies sind die offiziellen Empfehlungen des Robert-KochInstituts, die nicht nur für die Krankenhäuser, sondern für alle medizinischen Einrichtungen, also auch Praxen gelten“, so Dr. Caroline Herr aus München.

Das Screening auf MRSA erfordert Abstriche aus der Nase und dem Rachenraum, eventuell zusätzlich aus der Leiste und dem Analbereich. Bei Vorliegen offener Wunden wie beim diabetischen Fuß müssen auch dort Abstriche entnommen werden (Abb. 1).

\section{Sanierung von Wunden braucht Geduld}

Wird das Bakterium in der Nase oder im Rachen nachgewiesen, so muss der Patient isoliert werden. Für die Sanierung der Nase empfiehlt sich Mupirocin. Bei Rachenbefall sollte keine Mundspülung erfolgen, sondern immer eine systemische antibiotische Therapie mit Cotrimoxazol und Rifampicin eingeleitet werden. Der ausschließlich dermale MRSA-Befall ist sehr selten. Er erfordert eine antiseptische Körperwaschung, eine Isolierung solcher Patienten ist jedoch nicht erforderlich. Bei Befall des Anus bzw. Rektums ist eine antibiotische Therapie mit Vancomycin oral indiziert, jedoch keine Isolierung. „Ein besonders schwieriges Problem ist die Sanierung von Wunden“, so Höning. Dazu sei Zeit nötig. Systemische Antibiotika sind hier nicht die Mittel der ersten Wahl. Primär sei eine lokale Therapie ratsam, etwa mit einem silberhaltigen Präparat, Maden, Manuka-Honig-Produkten und Wundauflagen mit Polihexamid.

\section{Welche Antibiotika?}

Bei MRSA handele es sich zwar um nicht sehr virulente aber sehr anpassungsfähige Bakterien, die unter dem Druck der antibiotischen Therapie multiresistent geworden sind, sagte Höning. Deshalb sei es nötig, die obligatorische Antibiotikatherapie so zu gestalten, dass diese Bakterien keine Chance für neue Resistenzen haben. Eine antibiotische Kombinationstherapie sei immer indiziert, etwa der Standard Cotrimoxazol plus Rifampicin. Alternativ können Vancomycin, Fosfomycin oder Linezolid eingesetzt werden.

Dr. med. Peter Stiefelhagen

Quelle: Symposium beim Kongress „Diabetologie grenzenlos" am 11.2.2011 in München.

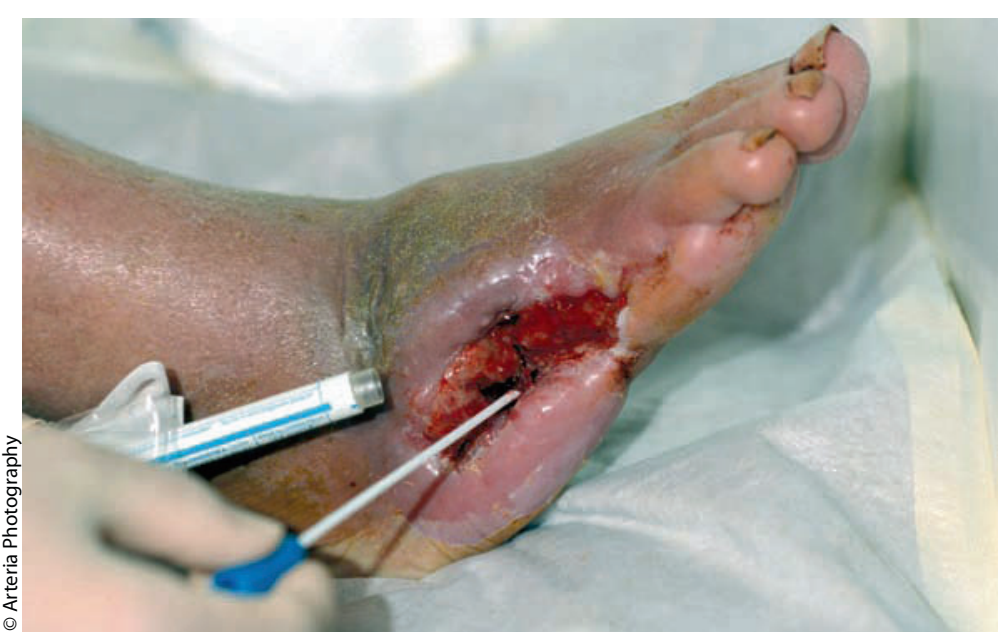

Abb. 1: Bei einem diabetischen Fuß sollte auch in Deutschland häufiger als bisher auf eine Besiedlung mit multirestistenten Staphylokokken gescreent werden. 